\title{
The politeness Package: Detecting Politeness in Natural Language
}

by Michael Yeomans, Alejandro Kantor, Dustin Tingley

\begin{abstract}
This package provides tools to extract politeness markers in English natural language. It also allows researchers to easily visualize and quantify politeness between groups of documents. This package combines and extends prior research on the linguistic markers of politeness (Brown and Levinson, 1987; Danescu-Niculescu-Mizil et al., 2013; Voigt et al., 2017). We demonstrate two applications for detecting politeness in natural language during consequential social interactionsdistributive negotiations, and speed dating.
\end{abstract}

\section{Introduction}

Politeness is a universal dimension of human communication (Goffman, 1967; Lakoff, 1973; Brown and Levinson, 1987). In practically all settings, a speaker can choose to be more or less polite to their audience, and this can have consequences for the speakers' social goals. Politeness is encoded in a discrete and rich set of linguistic markers that modify the information content of an utterance. Sometimes politeness is an act of commission (for example, saying "please" and "thank you") <and sometimes it is an act of omission (for example, declining to be contradictory). Furthermore, the mapping of politeness markers often varies by culture, by context (work vs. family vs. friends), by a speaker's characteristics (male vs. female), or the goals (buyer vs. seller). Many branches of social science might be interested in how (and when) people express politeness to one another, as one mechanism by which social co-ordination is achieved.

The politeness package is designed to make it easier to detect politeness in English natural language, by quantifying relevant characteristics of polite speech, and comparing them to other data about the speaker. For example, researchers may want to know whether politeness is associated with some situational or trait-level covariate (text as description). Or researchers might want to know whether people respond differently to polite rather than impolite language (text as treatment). Or they might want to know how an intervention affects the production of politeness (text as outcome). Finally, all of these analytical approaches can coalesce to support a theoretical model in which speakers strategically choose their politeness as a way to affect their audience's behavior (text as mediator).

Politeness typically draws from a common pool of linguistic markers for social co-ordination between speaker and listener. But the weight and valence of each marker depends on the context. Thus, we do not try to provide a single "politeness dictionary" for all contexts. Instead our approach draws from common methods in computational linguistics that use algorithms to select from a curated set of features. (Manning and Schütze, 1999; Grimmer and Stewart, 2013; Jurafsky and Martin, 2014). That is, we draw on existing linguistic theory to calculate a wide set of potentially relevant features from the text. But we then estimate the weights on those features empirically, defining politeness using some ground truth label—from a randomized treatment, or the listener, or a third party, or the speaker herself. We then use a supervised machine learning algorithm as a context-specific model of politeness: to classify unlabeled documents, and to characterize the nature of how politeness is expressed in the domain of interest.

Our software contributes to a rich ecosystem of general text analysis packages in $\mathrm{R}$. This includes structuring raw text (e.g. tidytext, tm, quanteda, coreNLP, spacyR), sentiment analysis (e.g., tidytext, SentimentAnalysis, syuzhet), and topic modeling (topicmodeling, stm). We incorporate some of these packages in our own work. Our package is the first in R to study politeness specifically, and also one of the first to focus on linguistic pragmatics more broadly. By focusing on the turn-level syntactic structure in natural language, our work is complementary to (and distinct from) existing work that primarily focuses on semantic content, such as identifying trends in topics or emotions over large corpora.

In this paper we also work through two important applications of politeness detection in social science. First, we measure manipulated politeness as a treatment effect in an experiment in which writers were instructed to write offers for a phone on craigslist. com, in a style that was high (or low) in politeness. This was used to validate a psychometric construct across several studies. Second, we measure observed politeness as a context-specific and naturally-occurring construct in a dataset on speed-dating. This was used to understand the meaning of politeness from different perspectives. 


\section{Politeness workflow}

The politeness package provides functions to identify politeness markers in natural language, graphically compare these to covariates of interest, develop a supervised model to detect politeness in new documents, and inspect high- and low-politeness documents. Table 1 summarizes the main functions of the politeness package. A full description of the functions is available in the package documentation.

\begin{tabular}{ll}
\hline Function & Description \\
\hline politeness() & $\begin{array}{l}\text { Detects linguistic markers of politeness in natural language. Takes an N-length } \\
\text { vector of text documents and returning an N-row data.frame of feature counts. } \\
\text { Some politeness features depend on grammatical parsing. }\end{array}$ \\
politenessPlot() & $\begin{array}{l}\text { Plots the prevalence of politeness features over a set of documents. Highlights } \\
\text { differences in politeness across covariate. }\end{array}$ \\
politenessProjection() & $\begin{array}{l}\text { Training and projecting a regression model of politeness based on a binary or } \\
\text { continuous variable. Supports both glmnet and textir, with LASSO as the default. } \\
\text { findPoliteTexts() }\end{array}$ \\
& $\begin{array}{l}\text { Finds examples of most or least polite texts in a corpus from a covariate identifying } \\
\text { politeness scores of texts. }\end{array}$ \\
\hline
\end{tabular}

Table 1: Politeness functions.

These tools can be combined in a workflow that we believe will be useful to most researchers interested in linguistic politeness. First, we offer a function, politeness () that will calculate a set of linguistic features that have been identified in the past as relating to politeness. Second, we offer a function, politenessPlot () to visualize these counts, in comparison to a covariate related to politeness (e.g., treatment/control). If the researcher wants to generate a politeness classifier, they can do so using the politenessProjection() function, which creates a single mapping from the politeness features in the supplied text to some other measure of interest. In particular, if the researcher has some "ground truth" labels of politeness over a set of texts, they can use this function as a politeness classifier, and automatically assign politeness scores to many more new texts.

\section{Politeness features}

Broadly speaking, the space of politeness features is guided by a rich literature on the linguistics of politeness (Goffman, 1967; Lakoff, 1973; Brown and Levinson, 1987). In general, politeness in language is designed to pay face to the listener, so that they feel respected. And while the linguistic markers of politeness vary from situation to situation, there are two common themes in most polite speech: Positive Politeness, and Negative Politeness.

Positive politeness involves actively bolstering the listener's self-image (showing gratitude, identifying as an in-group member, paying complements) as well as not derogating that image (complaints, cursing, informal titles, and so on). Negative Politeness involves respecting the listener's autonomy. This involves a general softening of statements, using hedges and adverbs. Requests may also be tempered, using indirect subjunctive language, and apologizing. Alternatively, speakers may express low negative politeness by making bare commands and being contradictory.

These elements are all included in this package as part of the politeness() function, which tallies 36 separate politeness markers (summarized in Table 2, along with examples of each.). Many are translated directly from recent research on the computational linguistics of politeness (DanescuNiculescu-Mizil et al., 2013; Voigt et al., 2017). We collected all of the features from these two papers, and removed a few that were very contextually specific (e.g., "keep your hands on the wheel" for drivers). However as we demonstrate below, many kinds of context-specific features can be helpful, and we show how to add them to the feature set manually.

The features in the politeness detector are summarized in in Table 2. We refer interested users to the original papers for details on the design of each feature. All features involve counting matches to a pre-defined list, which includes some combination of individual words, word stems, adjacency pairs, dependency pairs, part-of-speech-tags. Additionally, some features distinguish whether a match is found at the beginning of a sentence or not. Some (e.g., positive or negative emotion) include hundreds of possible matches; while others (e.g., "for you") are defined by a single phrase.

library (politeness)

data("feature_table") 


\begin{tabular}{|c|c|c|c|}
\hline Feature Name & POS Tags & Description & Example \\
\hline Hello & No & "hi", "hello", "hey" & "Hi, how are you today?" \\
\hline Goodbye & No & "goodbye", "bye", "see you later" & "That's my best offer. Bye!" \\
\hline Please Start & Yes & Please to start sentence & "Please let me know if that works" \\
\hline Please & Both & Please mid-sentence & "Let me know if that works, please" \\
\hline Gratitude & Both & "thank you", "i appreciate", etc. & "Thanks for your interest" \\
\hline Apologies & Both & "sorry", "oops", "excuse me", etc. & "I'm sorry for being so blunt" \\
\hline Formal Title & No & "sir", "madam", "mister", etc. & "Sir, that is quite an offer." \\
\hline Informal Title & No & "buddy", "chief", "boss", etc. & "Dude, that is quite an offer." \\
\hline Swearing & No & Vulgarity of all sorts & "The dang price is too high" \\
\hline Subjunctive & No & Indirect request & "Could you lower the price?" \\
\hline Indicative & No & Direct request & "Can you lower the price?" \\
\hline Bare Command & Yes & Unconjugated verb to start sentence & "Lower the price for me" \\
\hline Let Me Know & No & "let me know" & "Let me know if that works" \\
\hline Affirmation & Yes & Direct agreement at start of sentence & "Cool, that works for me" \\
\hline Conjunction Start & Yes & Begin sentence with conjunction & "And if that works for you" \\
\hline Reasoning & No & Explicit reference to reasons & "I want to explain my offer price" \\
\hline Reassurance & No & Minimizing other's problems & "Don't worry, we're still on track" \\
\hline Ask Agency & No & Request an action for self & "Let me step back for a minute" \\
\hline Give Agency & No & Suggest an action for other & "I want to let you come out ahead" \\
\hline Hedges & No & Indicators of uncertainty & "I might take the deal" \\
\hline Actually & Both & Indicators of certainty & "This is definitely a good idea." \\
\hline Positive & No & Positive emotion words & "that is a great deal" \\
\hline Negative & No & Negative emotion words & "that is a bad deal" \\
\hline Negation & No & Contradiction words & "This cannot be your best offer" \\
\hline Questions & No & Question words to start sentence & "Why did you settle on that value?" \\
\hline By The Way & No & "by the way" & "By the way, my old offer stands" \\
\hline Adverbial Just & Yes & modifying a quantity with "just" & "It is just enough to be worth it" \\
\hline Filler Pause & No & Filler words and verbal pauses & "That would be, um, fine" \\
\hline For Me & No & "for me" & "It would be great for me" \\
\hline For You & No & "for you" & "It would be great for you" \\
\hline First Person Plural & No & First-person plural pronouns & "it's a good deal for both of us" \\
\hline First Person Single & Both & First-person singular mid-sentence & "It would benefit me, as well" \\
\hline Second Person & Both & Second person mid-sentence & "It would benefit you, as well" \\
\hline First Person Start & Yes & First-person singular to start sentence & "I would take that deal" \\
\hline Second Person Start & Yes & Second-person to start sentence & "You should take that deal" \\
\hline Impersonal Pronoun & No & Non-person referents & "That is a deal" \\
\hline
\end{tabular}

Table 2: Politeness features detected by politeness(). Features that have "No" in the POS Tags column require part-of-speech (POS) tagging; where as those with "Both" can be approximated with out POS tagging, but POS tagging is recommended.

\section{Parsing grammar}

The meaning of a sentence often depends not just on its constituent words, but also on its grammatical structure. This is useful for words that can have different meanings, such as the adverbial "just" in "it is just enough" compared to the adjectival use in "the decision was just". Politeness can also be expressed in the grammatical structure itself (e.g., the unconjugated verbs in bare commands like "give me that!"). This information is lost in in bag-of-words analyses that do not label the sentence structure, or ignore word order.

As of this writing, there was no available grammar parsing software available wholly within the $\mathrm{R}$ language. Instead, we build off one of the most powerful natural language processing tools available currently - the Python module SpaCy (Honnibal and Johnson, 2015). It is open-source, fast, accurate, and well-benchmarked. We use spacyr (Benoit and Matsuo, 2017) to connect to SpaCy and take advantage of their pretrained models to identify the grammatical structure of each document. The current SpaCy model en_core_web_sm is a Convolutional Neural Network trained on OntoNotes, a large corpus comprising of news, conversational telephone speech, weblogs, usenet newsgroups, broadcast, and talk shows texts. Prior to using spacyr, users must install SpaCy in Python. As of spacyr version 0.9 .6 , this spacy installation can be detected automatically on the user's computer by spacyr when it is first called. ${ }^{1}$

\footnotetext{
${ }^{1}$ For advanced users, including those who may have multiple Python installations, you may have to initialize the SpaCy engine first, so that it is ready for use during the session. That is done using a separate function
} 
Many of the politeness features can be calculated without grammar parsing by setting parser="none". We recommend this as an initial first step for researchers, without having to install SpaCy. At this reduced setting, some features are dropped entirely (e.g., Bare Commands are a specific verb class). However, some features are approximated. For example, tags allow users to differentiate between a mid-sentence "please" and a "please" to start a sentence, while the tagless version of the function will collapse them into a single feature.

\section{Detecting politeness features}

The function politeness () takes in an $n$-length vector of texts, and returns an $n$-by- $f$ data.frame, with $f$ columns corresponding to the number of calculated features. We note that our package does not perform any spell- or grammar-checking on the text-though these kinds of errors can degrade the fidelity of the information in the text. Instead, we recommend that users do this on their own-either in $\mathrm{R}$, using another package like hunspell, or else using other software or by hand.

There are 36 features in total (see Table 2), but some user options affect the number of features that are returned. For one, if a part-of-speech tagger is not used (by setting parser = "none") then some features that depend on these tags will not be calculated, as detailed in Table 2. Additionally, you may use the default setting drop_blank = TRUE which will remove features that were not detected in any of the texts.

For example, consider the following texts.

library(politeness)

texts <- c("Hello, you", "You")

politeness() with parser="none" (default) will identify that both texts contain the feature Second.Person and that only the first contains Hello.

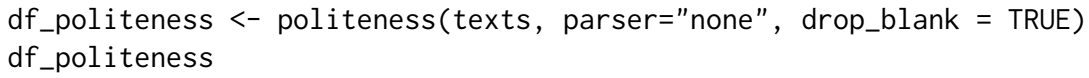

In order to get the full feature space we use parser="spacy". Now politeness() differentiates between the features Second.Person.Start (which captures the pronoun as a sentence subject) and Second.Person (which captures the pronoun as a sentence object) as shown below.

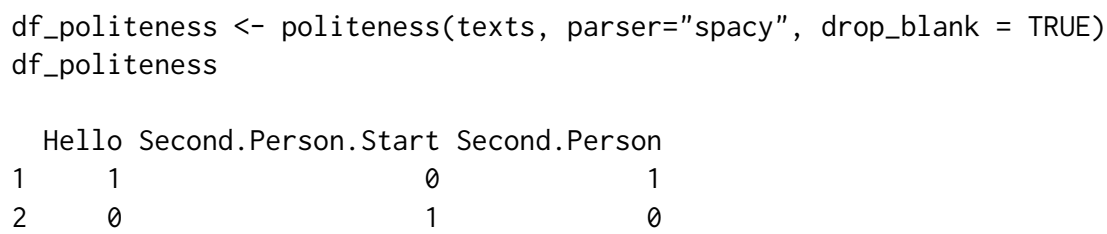

The examples above are very short, in practice longer sentences will use features multiple times (e.g., positive words). Setting metric="count" will populate each cell with the raw count of each feature in the text. Alternatively, metric="binary" will return a binary indicator of the presence or absence of each feature. Finally, metric="average" will count the prevalence of features as a percentage of the word count of each document. This is useful as a robustness check when there is wide variance in document length. For example take the following two texts (this data, borrowed from (Jeong et al., 2018) is included in the package):

data("phone_offers")

texts <- phone_offers\$message $[c(21,25)]$

texts

('spacyr: :spacy_initialize(python_executable = PYTHON_PATH)'-make sure to substitute in your preferred Python path name) and is explained well in the spacyr documentation. 
[1] "Hi I am very interested in your phone. It is exactly what I have been looking for. I would like to offer you 115 for it. It would make me very happy to buy the phone today."

[2] "Hi, I hope your day is going well. I am very pleased to see the phone you are offering for sale, as it is exactly what I need! I am on a very tight budget so I hope that you will be willing for accept $\$ 115$ for the phone. It is the most I can pay. Please know that I would be so happy if I am able to buy this phone. I'm sorry that I can't offer more. If you are willing to accept my offer, perhaps I can do you a small favor as well, like mow your lawn or something. In any case if you accept my offer you would have my sincere and heartfelt gratitude. Whether you accept my offer or not, I hope that this message finds you and yours well and happy. I hope you have a great day. :)"

Given a subset of features we observe the following counts.

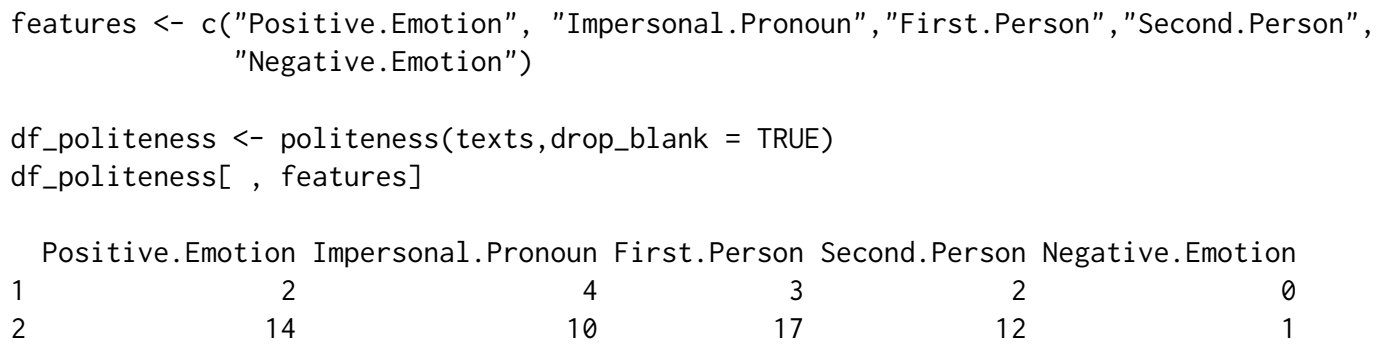

Although both texts have similar politeness features, the longer text contains larger count values. The option metric="binary", on the other hand, will return a simplified result with a 1 if the feature is present in the text as shown below.

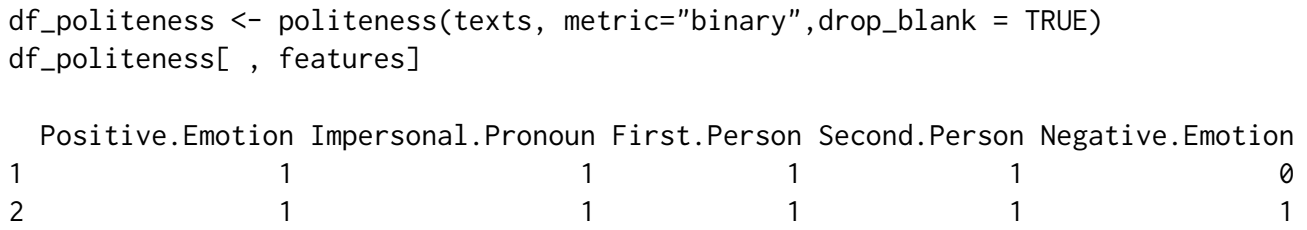

\section{Plotting politeness features}

The politenessPlot() function combines the politeness feature matrix with another measure of substantive interest that might covary with the prevalence of some of the politeness features-in particular, a ground-truth measure of politeness from annotation or assignment to treatment. This function generates a horizontal bar plot (with 95\% confidence intervals) of feature prevalence among two groups of documents.

The function can handle any kind of politeness metric (counts, binary, or averaged), but that distinction must be made in the initial call to politeness(). Below, we plot the politeness features from a feature count matrix in Figure 1, and a binary feature matrix in Figure 2.

The order of the bars themselves are sorted automatically, and determined by calculating the variance-weighted log odds of each feature with respect to the binary covariate. Many covariates are continuous. By default, this package treats the top and bottom terciles of that distribution as binary categories, with the middle tercile dropped. Users can also create their own categories beforehand, and enter those labels in place of the covariate.

Often some features are not meaningful for further analysis - either because they are too rare in the data, or else because they do not meaningfully covary with the covariate of interest. Users have two options to exclude these from the plot. First, the drop_blank parameter can remove rare features it takes a number between 0 and 1, which determines a cut-off based on prevalence. Specifically, all features which appear in less than this proportion of texts are excluded from the plot. To include all features, leave this value at 0 . 
Second, the middle_out parameter can remove features which do not vary meaningfully across the covariate. Each feature is evaluated using a two-sample t.test, and features are removed when the p-value of this test lies above the user's provided cut-off, a number between 0 and 1 (the default is 0.1 ). To include all features, simply set this value at 1 .

df_politeness_count <- politeness(phone_offers\$message, parser="none", drop_blank=FALSE)

politenessPlot(df_politeness_count, split=phone_offers $\$$ condition, split_levels = c("Tough", "Warm"), split_name $=$ "Condition")

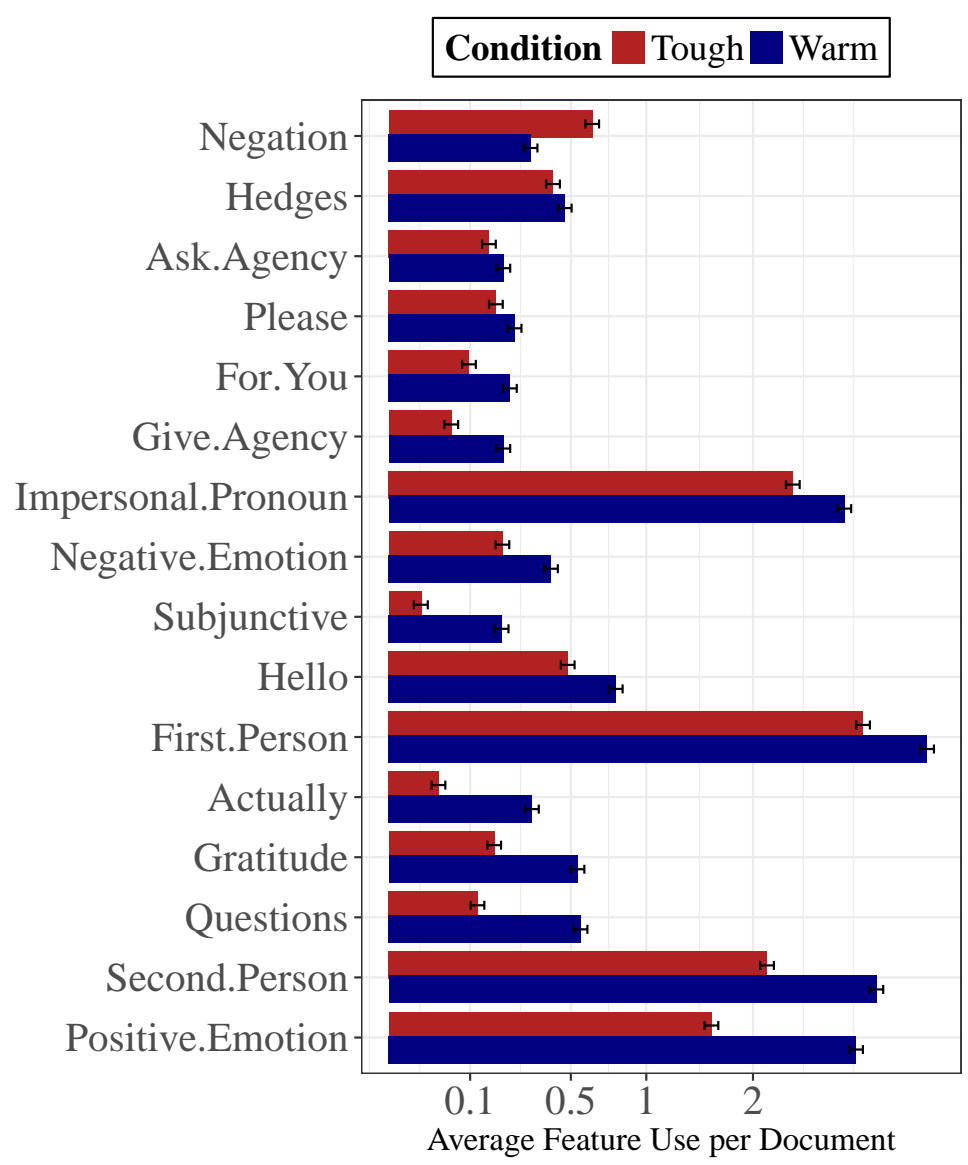

Figure 1: Politeness plot showing for each feature the average use per document in square root scale. Features are ordered by their variance-weighted log odds. This can accommodate any metric for the politeness features (count, binary, average) but that must be set in the call of politeness() before the data.frame is passed the plot function.

df_politeness_binary <- politeness(phone_offers\$message,

$$
\begin{aligned}
& \text { parser="none", } \\
& \text { metric="binary", } \\
& \text { drop_blank=FALSE) }
\end{aligned}
$$

politenessPlot(df_politeness_binary, split=phone_offers\$condition, split_levels = c("Tough", "Warm"), split_name = "Condition") 


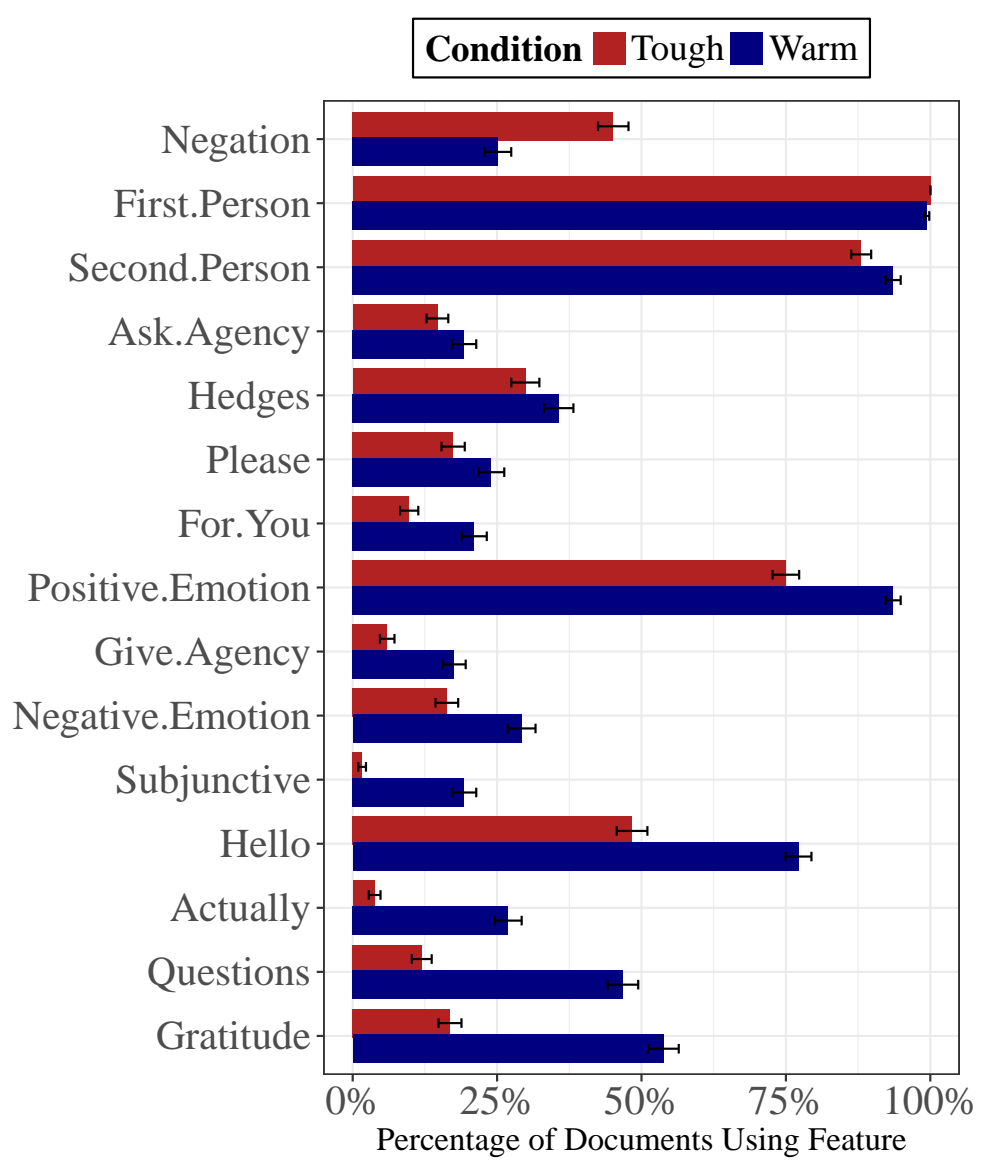

Figure 2: Politeness plot showing percentage of documents showing each feature. Features are ordered by their variance-weighted log odds. In order to plot the percentage of documents showing each feature metric="binary" must be set in the call of politeness () .

\section{Projecting politeness features}

Users can generate a politeness classifier with the politenessProjection() function. This creates a single mapping from the politeness features in the supplied text to the covariate of interest. This can then be used to predict the covariate itself in held-out texts. In particular, if the user has some "ground truth" labels of politeness over a set of texts, they can use this function as a politeness classifier, and automatically assign politeness scores to many more new texts. This ground truth can be labelled by annotators in observational data, or, as in our example below, generated from a randomized experiment in which the text itself is the outcome.

This function is a wrapper around supervised learning algorithms. The default uses glmnet (Friedman et al., 2010), the vanilla LASSO implementation in R. We also allow users to use a different algorithm, textir (Taddy, 2013), which implements a massively multinomial inverse regression. Intuitively, this model represents a more realistic causal structure to text-plus-covariate data - that is, the metadata is typically an ex ante property of the speaker that has a causal effect on the words they use, rather than the words having a causal effect on the speaker's metadata. Both packages have their merits, though for now we recommend using glmnet to start, especially if its use is familiar.

In addition to the phone_offers dataset, we have included a smaller bowl_offers dataset (also from (Jeong et al., 2018)). Participants in this study were given similar instructions (i.e., communicate in a warm or tough style) but for a different negotiation exercise. We use the phone_offers dataset to define the construct of interest, and use the bowl_offers dataset as held-out data in politenessProjection(). The results confirm that the manipulation in the held-out data induced more politeness in one condition than the other.

In addition to the projected labels for the new documents, politenessProjection() also returns the coefficients estimated in the model. This provides some transparency regarding the exact functional form of politeness being generated. However, we caution users that the coefficients are chosen to maximize the prediction accuracy of the model of the whole, rather than recovering the "true" 
coefficient for any particular feature. In particular, these models may not reflect the first-order relationships in the data, especially when many relevant features are colinear with each other.

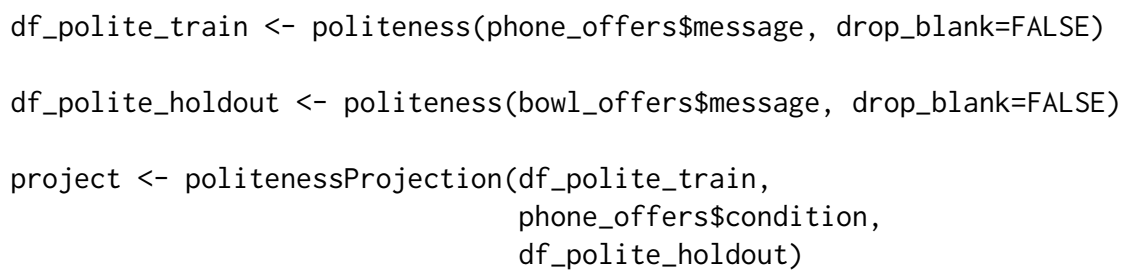

$\begin{array}{rr}\text { Negation } & \text { Informal.Title } \\ -1.04272459 & 0.10637694 \\ \text { Give.Agency } & \text { Hello } \\ 0.48516687 & 0.60900150 \\ \text { Gratitude } & \text { Actually } \\ 0.69019865 & 1.34960715 \\ \text { Second.Person } & \\ 0.03484301 & \end{array}$

We consistently recommend that researchers build a context-specific model of politeness for their own datasets, using labeled examples from within-domain. However, some new users may want to try this workflow before deciding whether to hand-label new documents in their own domain. In that case, users can follow the example above to build a rudimentary out-of-the-box politeness classifier with the phone_offers dataset and the politenessProjection() function). This analysis assumes that the rules of politeness in the user's domain are identical to the rules of politeness in negotiations, which may or may not hold.

\section{Finding examples of polite and impolite documents}

Before users apply any output from the politenessProjection() function to other analyses, they should first be curious about examples of texts that best represent the distinction made by that projection (i.e., the most- or least-typically polite texts()). The findPoliteTexts() function replicates the analyses of politenessProjection but instead returns a selection of the texts that are the most extreme (i.e., high, or low, or both) along the projected dimension. The parameters type and num_docs allow users to specify the type and number of texts that are returned.

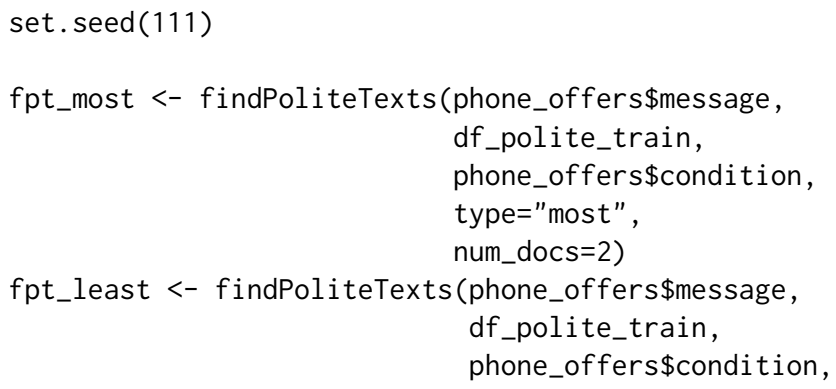


type="least",

num_docs=2)

fpt_most\$text

[1] Hello, Oh my goodness, I'm so excited to see your listing. The phone is EXACTLY what I have been searching for! And I can tell from your description and the photo that it's just perfect, too. I can almost hear it ringing in my pocket right now! What's that? Hello? So, happy to hear from you. Sorry, sorry. I'm getting ahead of myself.

Soooo, I was wondering if there was any way that you might consider taking a little bit less for this phone? It's absolutely everything I've been looking for but my bosses \&lt; grrrrr!!!\&gt; will only give us $\$ 115$ to get this phone. I know! Can you believe it? Is there any way you could absolutely make my day and say " "yes" " to \$115? I'd be so, so grateful. Just let me know when you can. Thanks so much. :)

[2] Hello, I am glad you're selling this phone. Is it still available? I would love to purchase it. Would you consider $\$ 115$ for it? I can buy it today if that price works. Thank you.

fpt_least\$text

[1] I am inquiring about your Iphone 6 plus that you had posted. I am wanting to buy and I have cash in hand. The max amount I can offer is $\$ 115$. No more, no less.

Let me know.

[2] Come on. The price you are offering on a product that ISN'T NEW is unreasonable. Now, I for one am very interested in getting this item. BUT, I will only pay $\$ 115$. I am not paying a penny more.

\section{Execution time}

In principle, these functions can handle an arbitrarily large set of documents. In practice, however, language data can be quite large, both in the amount of documents and the length of the documents. This can have a marked effect on execution time, especially on smaller computers.

In order to reduce computation time, we use data.table (Dowle and Srinivasan, 2017) and quanteda (Benoit et al., 2018) in the backend which have fast implementations of data manipulation. We also analyzed the execution time of intermediate steps within politeness() to identify any potential critical points.

To provide rough benchmarks, we ran the politeness () function with a range of document counts $\left(10^{2}, 10^{3}, 10^{4}\right)$ and lengths $(100,200)$. The tests were performed using a 2012 Macbook Pro with a 2.5 $\mathrm{GHz}$ Intel Core i5. For each case we ran it three times, both with and without part-of-speech tagging, and the resulting execution times are plotted below.

Figure 3 shows that poli teness() scales reasonably well for large sets of documents. For example, given 200-word documents, and using the spacy parser, we found that 100 and 1,000 length vectors of texts take an average of 7.3 and 70.6 seconds, respectively. We recommend that for larger corpora, researchers test the code on smaller subsets first.

A common and complex situation is when text is broken up into many documents per observation. One example of this would be a conversation transcript, in which people take many turns over a single conversation, but the covariates are measured at the level of the person, or conversation. In these cases, we recommend calculating the politeness markers in individual documents separately, and then aggregated into person-level counts afterwards, for plotting, analysis, and model-building. This will be more efficient and more accurate than concatenating each person's turns into one long document and calculating the politeness of the entire text at once. 


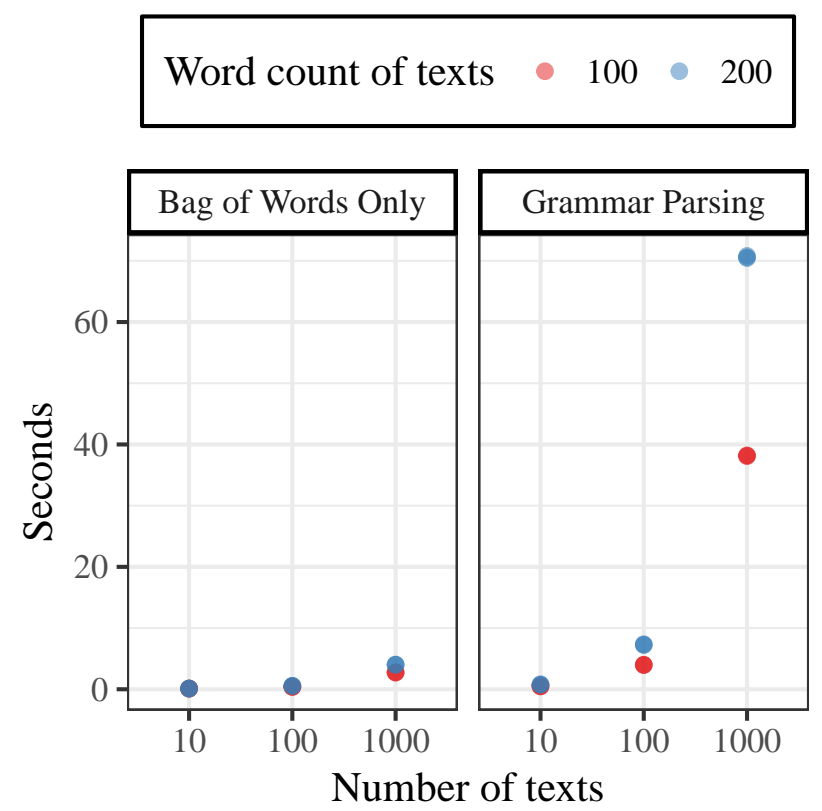

Figure 3: Execution time of politeness() with different number of texts, length of texts, and parsers.

\section{Extended Example: Politeness in Speed Dating}

We demonstrate the broader applications of our package in a new context: courtship, in face-to-face conversation with many turns per speaker. This example comes from the SpeedDate corpus (originally published in (Ranganath et al., 2009; McFarland et al., 2013). Here, politeness is not a treatment effect. Instead, we model the naturally-occurring variation in politeness (as rated by listeners) during a series of speed dating events. Furthermore, we highlight the context-specificity of politeness-we compare the linguistic markers of politeness in both female speakers (as rated by men) and male speakers (as rated by women), while holding constant the domain, the outcome measure, and the conversation itself.

The data were collected over three evenings, in which 110 heterosexual participants each met with 15-20 other potential partners for four minutes at a time. The main observations are gathered from each dater, from a survey immediately after their date. For privacy reasons, we cannot include the text of the dates themselves. However, we do include the matrix of politeness features as calculated by the politeness() function.

Note that the original function call treats each turn in the dataset as a separate document, to produce a one-row-per-turn matrix. So to analyze these data within our framework we need to condense the politeness feature matrix to the same one-row-per-person-date level. To do this here, we loop through each row of the per-person-date data, and add up the counts from the relevant section of the per-turn database.

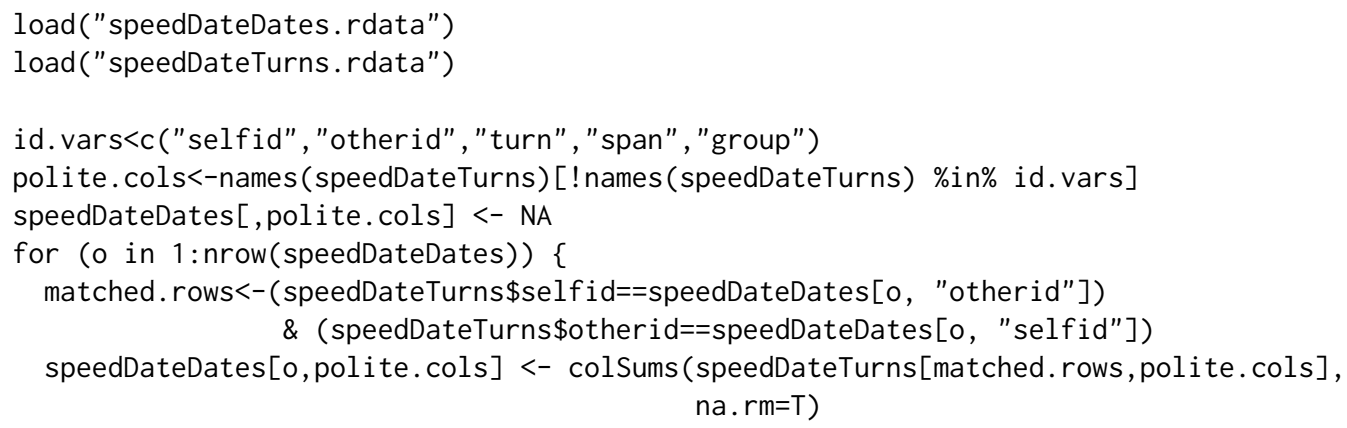

We wanted to include extra domain-specific features, to capture elements of live face-to-face conversation. These were calculated separately, and merged into the politeness feature matrix. First, we counted moments of laughter, which were indicated by the transcribers. We also included repair questions (e.g., "pardon?") using a list from Ranganath et al. (2009). Finally, we included four kinds 


$\begin{array}{lllllll} & \text { Followup.Qs } & \text { Switch.Qs } & \text { Intro.Qs } & \text { Mirror.Qs } & \text { Repair.Qs } & \text { Laughter } \\ \text { Spoken by Women } & 4.04 & 2.89 & 0.26 & 1.85 & 0.13 & 4.42 \\ \text { Spoken by Men } & 5.11 & 3.51 & 0.38 & 1.73 & 0.17 & 1.83\end{array}$

Table 3: Prevalence of features added to politeness detector, by gender.

of question types from Huang et al. (2017)—switch questions, which change the topic; follow-up questions, which expand on the current topic; introductory questions, which open a dialogue; and mirror questions, in which one person returns a question that they had just been asked. By merging these six extra features into the politeness feature matrix, downstream functions will treat them as though they were part of the original feature set.

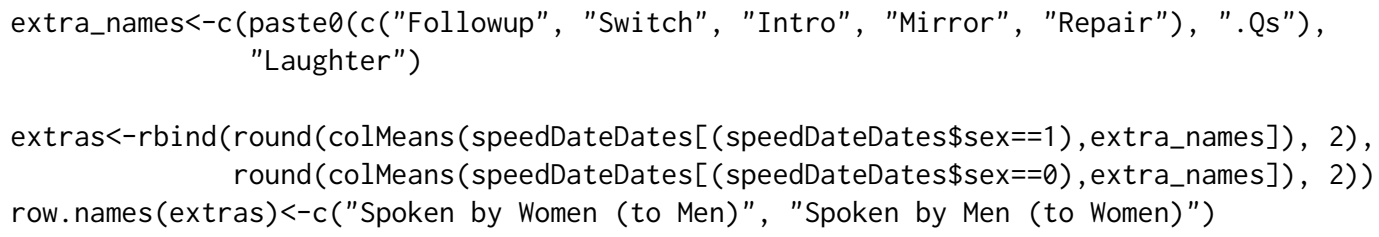

extras

Immediately after each date, both participants evaluated their partner on a set of dimensions. The one most relevant for our purposes was the question "how courteous was your partner", which was rated on a scale from 1-10 (plotted in Figure 4). We use this as a ground truth measure of politeness. Because politeness was measured (not manipulated) we must dichotomize the continuous variable into two groups. We drop the middle third of medium-courtesy dates and compare the dates that were rated as most and least rude, to increase contrast. For clarity we do this manually in the example below, but the package also does this automatically if the provided labels are on a continuous scale.

speedDateDates\$politeness <- " "

speedDateDates\$politeness[speedDateDates\$courtesy > 8] <- "Courteous"

speedDateDates\$politeness[speedDateDates\$courtesy < 6] <- "Rude"

speedDateDates<-speedDateDates[speedDateDates\$politeness != " , ]

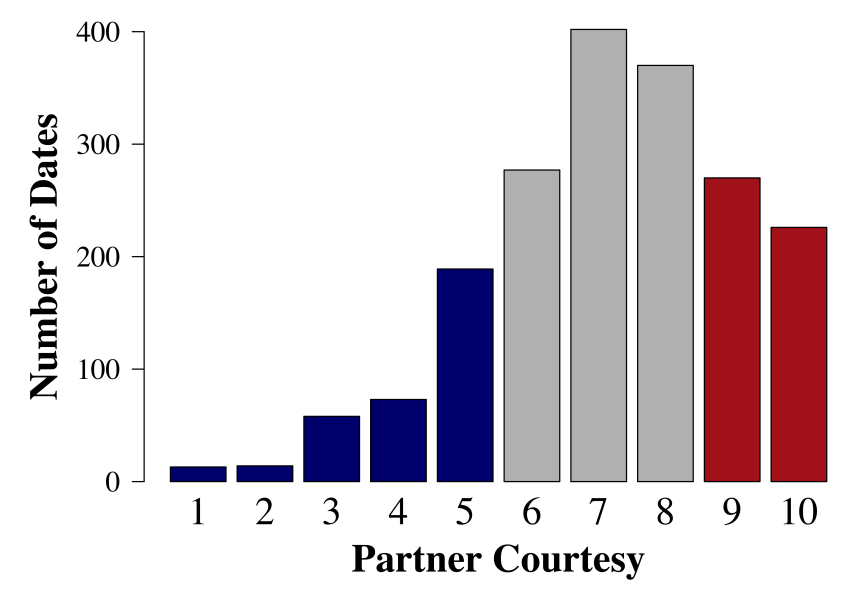

Figure 4: Distribution of rated courtesy in SpeedDate corpus.

Finally, we wanted to know whether the rules by which politeness is judged were different for men and for women. The average courtesy rating of men by women was slightly lower than that of the women by men. But these summary ratings do not tell us anything about how rudeness or courtesy is determined. Instead, we apply the politenessPlot() function among each gender separately to determine gender-specific models for how politeness is related to the linguistic choices of the speaker.

politenessPlot (speedDateDates[(speedDateDates $\$$ sex==1), 
$c$ (polite.cols, extra_names)],

split=speedDateDates $\$$ poli teness [ (speedDateDates $\$$ sex $==1)]$, split_name="Ratings of Women (by Men)",

top_title="",

middle_out $=0.1$ )

politenessPlot (speedDateDates $[($ speedDateDates $\$$ sex $==0$ ),

c(polite.cols, extra_names)],

split=speedDateDates $\$$ politeness $[($ speedDateDates $\$$ sex $==0)]$, split_name="Ratings of Men (by Women)",

top_title $="$ ",

middle_out $=0.1$ )
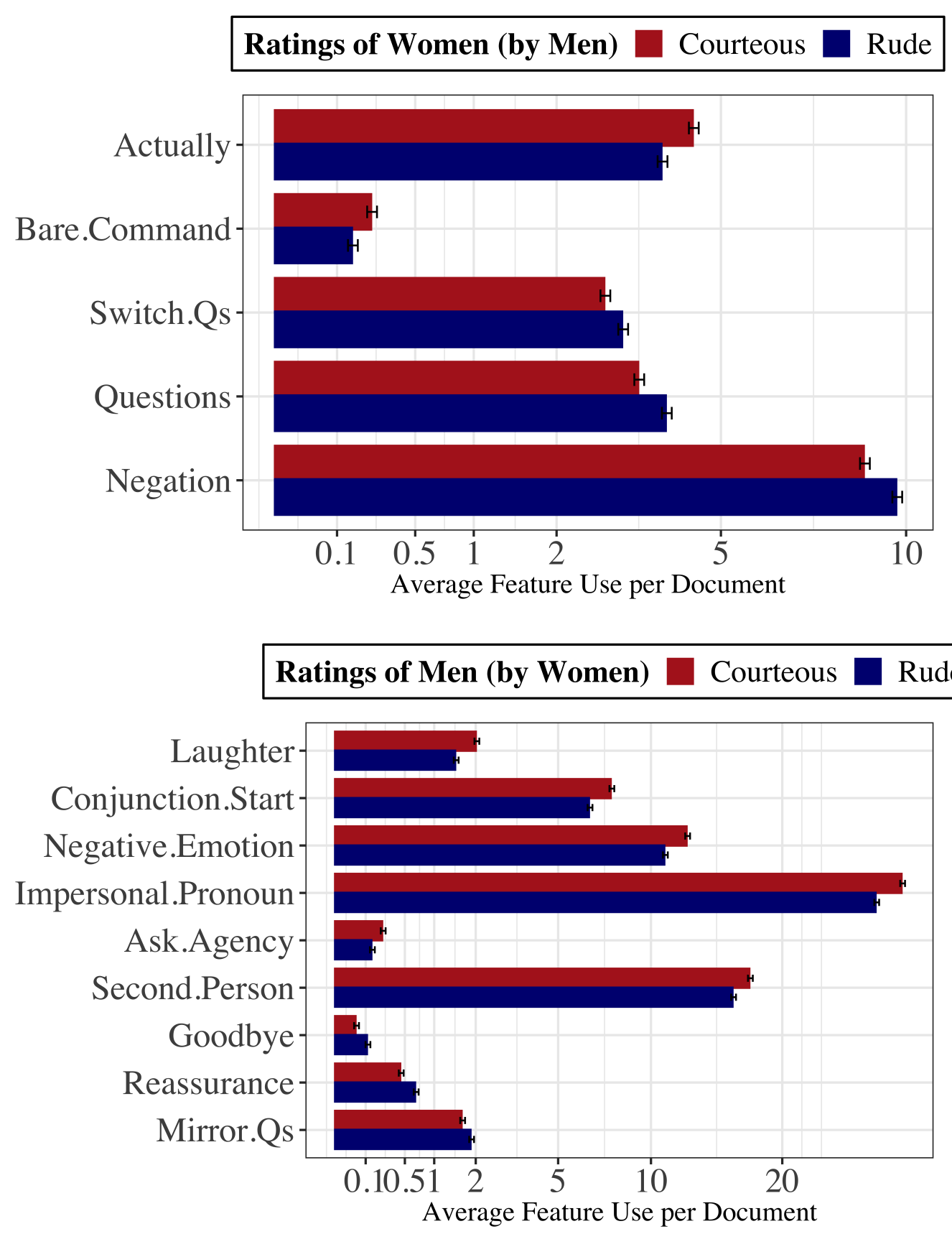

Figure 5: Politeness markers in speed-dating by speaker gender. 
The results in Figure 5 demonstrate that the rules for what is considered polite behavior from a man or a woman can be quite different, even the same conversation. Men laugh less often than women, overall; but when they do laugh it is associated with courtesy by their partner. Men who are considered rude tend to give formal goodbyes as they are leaving, and more often respond to a question by mirroring it back to their partner. On the other hand, women are seen as rude when they contradict, or when they ask questions (in particular, topic-switching questions) but are seen as polite when they reveal more about themselves-the "actually" feature captures pivots to greater detail, such as "in fact" or "to be honest." Interpreting these features can be difficult without the text in hand, though, and users are encouraged to incorporate qualitative analyses of their own data, in addition to the analyses provided here.

We note that the differences in feature use between polite and impolite daters (of both genders) are not as stark in this example as in the negotiation study. This is for two reasons. First, the variation in politeness is naturally occurring, rather than induced by a randomized treatment. Contrasts will be greater when writers are instructed and able to adopt a particular communication style. Secondly, the speed daters communicated face-to-face, so there could be other sources of information that would affect the listener's rating of a speaker's politeness (such as intonation, body language, pacing, and so on). In datasets where they are measured, these other features could be added to the workflow in the same way as the extra text-based features above.

\section{Conclusions}

We detail an empirical model of politeness, as codified in the politeness package. We use simple examples to show the range of output from the politeness() and politenessPlot() functions. We also show how politenessProjection() can be used to develop a domain-specific supervised model for politeness, which can be used by findPoliteTexts() to identify and explore distinctive documents. We also work through two examples of consequential politeness-as manipulated amongst negotiators, and as observed amongst speed daters-that highlight many challenges that are common for all sorts of research in $\mathrm{R}$ that uses natural language data from social interactions.

The tools presented here should be useful for all researchers who study how people interact with one another, across a wide variety of contexts. In future work, we hope to expand this toolkit to handle a wider range of politeness markers that are not present in proper written english (including shorthand, slang, nonverbals, and other languages). Further research is also needed into the extent to which the markers of politeness can vary from one context to another, and the consequences of politeness on interpersonal relations.

\section{Acknowledgements}

We thank the Spencer Foundation, the Hewlett Foundation, Harvard's Institute for Quantitative Social Science, and the Harvard Vice Provost for Advances in Learning Research Group for their support.

\section{Bibliography}

K. Benoit and A. Matsuo. spacyr: Wrapper to the 'spaCy' 'NLP' Library, 2017. URL https: //CRAN. Rproject. org/package=spacyr. R package version 0.9.2. [p491]

K. Benoit, K. Watanabe, H. Wang, P. Nulty, A. Obeng, S. Müller, and A. Matsuo. quanteda: An r package for the quantitative analysis of textual data. Journal of Open Source Software, 3(30):774, 2018. doi: 10.21105/joss.00774. URL https: //quanteda.io. [p497]

P. Brown and S. C. Levinson. Politeness: Some universals in language usage. Studies in interactional sociolinguistics, 4, 1987. URL http://psycnet.apa.org/record/1987-97641-000. [p489, 490]

C. Danescu-Niculescu-Mizil, M. Sudhof, D. Jurafsky, J. Leskovec, and C. Potts. A computational approach to politeness with application to social factors. CoRR, abs/1306.6078, 2013. URL http: //arxiv.org/abs/1306.6078. [p489, 490]

M. Dowle and A. Srinivasan. Data.table: Extension of 'data.frame', 2017. URL https://CRAN. R-project. org/package=data. table. R package version 1.10.4-3. [p497]

J. Friedman, T. Hastie, and R. Tibshirani. Regularization paths for generalized linear models via coordinate descent. Journal of Statistical Software, 33(1):1-22, 2010. URL http: / /www. jstatsoft. org/ v33/i01/. [p495] 
E. Goffman. On face-work. Interaction Rituals, pages 5-45, 1967. [p489, 490]

J. Grimmer and B. Stewart. Text as data: The promise and pitfalls of automatic content analysis. Political Analysis, 21(3):267-297, 2013. [p489]

M. Honnibal and M. Johnson. An improved non-monotonic transition system for dependency parsing. In Proceedings of the 2015 Conference on Empirical Methods in Natural Language Processing, pages 13731378, Lisbon, Portugal, 2015. Association for Computational Linguistics. URL https: //aclweb . org/ anthology/D/D15/D15-1162. [p491]

K. Huang, M. Yeomans, A. W. Brooks, J. Minson, and F. Gino. It doesn't hurt to ask: Question-asking increases liking. Journal of Personality and Social Psychology, 113(3):430-452, 2017. [p499]

M. Jeong, J. Minson, M. Yeomans, and F. Gino. Communicating warmth in distributive negotiations is surprisingly counter-productive. Working Paper, 2018. [p492, 495]

D. Jurafsky and J. Martin. Speech and Language Processing. Pearson, 2014. [p489]

R. T. Lakoff. The Logic of Politeness: Minding Your P'S and Q's. Chicago Linguistic Society, 1973. URL https://books.google.com/books?id=DfWfNAAACAAJ. [p489, 490]

C. Manning and H. Schütze. Foundations of Statistical Natural Language Processing. MIT Press, 1999. [p489]

D. McFarland, D. Jurafsky, and R. Ranganath. Making the connection: Social bonding in courtship situations. American Journal of Sociology, 118(6):1596-1649, 2013. [p498]

R. Ranganath, D. Jurafsky, and D. McFarland. It's not you, it's me: Detecting flirting and its misperception in speed-dates. Proceedings of the 2009 Conference on Empirical Methods in Natural Language Processing, 1(1):334-342, 2009. [p498]

M. Taddy. Multinomial inverse regression for text analysis. Journal of the American Statistical Association, 108(503):755-770, 2013. doi: 10.1080/01621459.2012.734168. URL https://doi . org/10.1080/ 01621459.2012.734168. [p495]

R. Voigt, N. P. Camp, V. Prabhakaran, W. L. Hamilton, R. C. Hetey, C. M. Griffiths, D. Jurgens, D. Jurafsky, and J. L. Eberhardt. Language from police body camera footage shows racial disparities in officer respect. Proceedings of the National Academy of Sciences, 114(25):6521-6526, 2017. ISSN 0027-8424. URL https://doi .org/10.1073/pnas.1702413114. [p489, 490]

Michael Yeomans

Harvard University

Cambridge, MA 02138, USA

yeomans@fas.harvard.edu

Alejandro Kantor

Institute for Quantitative Social Science

Harvard University

Cambridge, MA 02138, USA

alejandrokantor@fas. harvard. edu

Dustin Tingley

Department of Government

Harvard University

Cambridge, MA 02138, USA

dtingley@gov. harvard. edu 